\title{
Determining the Factors that Drive Twitter Engagement-Rates
}

\author{
Gulsah Semiz \\ Bentley University \\ Waltham, MA 02452 \\ U.S.A. \\ Paul D. Berger \\ Bentley University \\ Waltham, MA 02452 \\ U.S.A.
}

\begin{abstract}
Twitter is one of the most important social networks and is a micro-blogging site where users send short messages, often called, "tweets." In this paper, we explore the determination of variables that affect the engagement rate of a tweet. We utilize data during the first 11 months of 2016 from TurkishWIN's Twitter Handle, and use Excel and SPSS to analyze several variables with respect to their impact on engagement rate. These variables includes the day of the week, the time of day, the length of the tweet, the number of mentions, the number of hashtags, and the language of the tweet among other variables. Using stepwise regression analysis, we find that several variables have a highly significant relationship to the engagement rate of a tweet. We discuss each significant variable in terms of the exact relationship it has with tweet engagement, and the implications of these relationships. Lastly, we note the limitations of our study and suggested directions for future research.
\end{abstract}

KeyWords: Twitter, Tweet, Engagement-Rate, Hashtags, Mentions, Stepwise regression.

\section{INTRODUCTION}

The way brands interact and engage with their audience has changed significantly with the use of social media. Today, brands are present in several different social networks and each has a different definition of success criteria in order to measure the engagement between brands and target audiences.

Twitter is one of the most popular social networks and also is a micro-blogging site in which users are allowed to send short messages, what are called "tweets," of up to 140 characters. Twitter has 317 million monthly active users on average as of the third quarter of 2016 [1]. Therefore, it's one of the important social networks where people, companies, and non-profits can make their voice heard. There are several metrics collected by Twitter which show different types of attributes that users can interact with, and engage with, a tweet. We will consider a number of them.

This paper aims to determine the most important variables that affect the engagement rate of a tweet. The engagement rate is defined as the number of engagements divided by the number of impressions; in other words, it indicates the proportion (or, if multiplied by 100, the percentage) of the people who saw the tweet who, indeed, engaged with it. Since the core 
value-proposition of social networks is creating an interaction among users, the engagement rate on Twitter is a metric that illustrates how well the content resonates with the people who see it (i.e., the targeted audience).

\section{METHODOLOGY}

The data used in these analyses were exported directly from TurkishWIN's Twitter Handle [2], edited on Excel and utilized on SPSS. The time period of the data is from January 1, 2016 to November 30, 2016, and the data contains 1,489 tweets along with metrics such as impressions, engagements, engagement rate, profile clicks, media engagements and so on (all to be defined later), for each tweet. Although the most important determinant of the engagement rate is likely the message, itself (i.e., the content,) there may be other variables that potentially are related to, and thus, predict, the engagement rate.

Following are the "independent variables" that we consider as possibly associating with the engagement rate:

- $\quad$ The number of hashtags - see definition in Table 1.

- The number of handles mentioned (i.e., "mentions") - see definition in Table 1.

- The time of the day the tweet was sent; 3 categories: Morning; Afternoon, Evening.

- The day of the week the tweet was sent; 7 categories: Monday, Tuesday, Wednesday, and so on.

- Including a medium, such as video or image; 2 categories: Yes, No

- The length of the tweet text without counting the URLs (24 character per each). - see definition in Table 1.

- Including a link (URL) of another site; 2 categories: Yes, No

- The main language used in the tweet; 2 categories: English, Turkish. 
Table 1 defines general terms used by Twitter and in this paper.

Table 1: Definition of General Terms*

- Username: A username, or a Twitter handle, is how users are identified on Twitter, and is always preceded immediately by the @ symbol. For instance, Katy Perry is @katyperry.

- Follower: A follower is another Twitter user who has followed someone to receive his/her Tweets in the Twitter feed.

- Hashtag: A hashtag is any word or phrase immediately preceded by the \# symbol. When you click on a hashtag, you'll see other Tweets containing the same keyword or topic.

- Like (n.): Liking a Tweet indicates that you appreciate it. You can find all of your likes by clicking the likes tab on your profile. Tap the heart icon to like a Tweet.

- Mention: Mentioning other users in your Tweet by including the @ sign followed directly by their username is called a "mention." Also, refers to Tweets in which your @username was included.

- Re-tweet (v.): The act of sharing another user's Tweet to all of your followers by clicking on the Re-tweet button.

- Tweet (n.): A Tweet may contain photos, videos, links and up to 140 characters of text.

- Tweet (v.): The act of sending a Tweet. Tweets get shown in Twitter timelines or are embedded in websites and blogs.

- URL, URLs: A URL (Uniform Resource Locator) is a web address that points to a unique page on the internet.

\section{Source of the definitions: [3] https://support.twitter.com/articles/166337\#}

Since Twitter-exports include only the basic metrics - impressions, clicks, engagements etc., along with the tweet text, we need to create some of the variables listed above, that are not present directly. In addition, we need to define selected variables in a way that is amenable to having them appropriately represented in a multiple regression analysis.

The date and time of the tweet is present in the Twitter export. The number of hashtags, the number of mentions, and the number of links that each tweet has, as well as the length of the tweet text, are analyzed from the tweet-text via Excel, by using the IF, COUNTIF, SUBSTITUTE, and LEN formulas. All of these variables are ratio-scale (i.e., interval scale, along with having a true zero point.)

The variables such as the day of the week and the time of the day the tweet was sent, whether the tweet included a medium or not, the language used in the tweet, and whether the tweet 
included a link to another site, are nominal scale variables. Dummy variables were constructed to represent each variable in a regression analysis; all were created as $(0,1)$ variables. Table 2 contains a list of the variables eligible in the stepwise-regression analysis.

Table 2: A list of variables eligible for the stepwise-regression analysis (variable name in bold)

\begin{tabular}{|c|c|}
\hline Variables & Definition \\
\hline The day of the week - Sunday & 1 if Sunday, 0 otherwise \\
\hline The day of the week - Monday & 1 if Monday, 0 otherwise \\
\hline The day of the week - Tuesday & 1 if Tuesday, 0 otherwise \\
\hline The day of the week - Wednesday & 1 if Wednesday, 0 otherwise \\
\hline The day of the week - Thursday & 1 if Thursday, 0 otherwise \\
\hline The day of the week - Friday & 1 if Friday, 0 otherwise \\
\hline The day of the week - Saturday & 1 if Saturday, 0 otherwise \\
\hline The time of the day - Morning (6:00am to 11:59am) & 1 if Morning, 0 otherwise \\
\hline The time of the day - Afternoon (12:00pm to 5:59pm) & 1 if Afternoon, 0 otherwise \\
\hline $\begin{array}{l}\text { The time of the day - Evening (6:00pm to } 11: 59 \mathrm{pm}) \\
\text { (Tweets from 12:00am to 5:59am are excluded from } \\
\text { the data set) }\end{array}$ & 1 if Evening, 0 otherwise \\
\hline The Length of the tweet without URLs & Actual value \\
\hline The number of Mentions & Actual value \\
\hline The number of Hashtags & Actual value \\
\hline Medium & $\begin{array}{l}1 \text { if Medium included, } 0 \\
\text { otherwise }\end{array}$ \\
\hline Link (to another site) & $\begin{array}{l}1 \text { if Link to another site is } \\
\text { present, } 0 \text { otherwise }\end{array}$ \\
\hline $\begin{array}{l}\text { Language (of the tweet) } \\
\quad \text { (Tweets in other languages excluded from data set) }\end{array}$ & 1 if English, 0 if Turkish; \\
\hline
\end{tabular}

It should be noted that, since we are performing a stepwise-regression analysis, and not a "regular" multiple-regression analysis, there is no need to limit the number of dummy variables in a set to one fewer than the number of categories being captured. For two-category variables, we have created one dummy variable; however, for the variables with more than two categories (i.e., day of the week tweet sent, time of the day tweet sent), we have included a variable of each category.

In this research, we are trying to determine the best model to predict the engagement rate. Therefore, we have performed a stepwise regression. The stepwise regression contains engagement rate as the dependent ("output") variable and the 16 variables listed in Table 2 as potential independent variables. We used the default settings on SPSS, with the "p-value to enter" $=.05$, and the "p-value to delete" $=.10$. Table 3 provides the official definition of engagement rate, along with selected other metrics that Twitter has defined. 
Table 3: Definition of a variety of Twitter metrics, including Engagement Rate.

- Detail expands: Clicks on the Tweet to view more details

- Embedded media clicks: Clicks to view a photo or video in the Tweet

- Engagements: Total number of times a user interacted with a Tweet. Clicks anywhere on the Tweet, including Retweets, replies, follows, likes, links, cards, hashtags, embedded media, username, profile photo, or Tweet expansion

- Engagement rate: Number of engagements divided by impressions

- Follows: Times a user followed you directly from the Tweet

- Hashtag clicks: Clicks on hashtag(s) in the Tweet

- Impressions: Times a user is served a Tweet in timeline or search results

- Likes: Times a user liked the Tweet

- Link clicks: Clicks on a URL or Card in the Tweet

- Replies: Times a user replied to the Tweet

- Retweets: Times a user retweeted the Tweet

- Shared via email: Times a user emailed the Tweet to someone

Source of the definitions: [4] https://support.twitter.com/articles/20171990\#

ANALYSIS AND DISCUSSION OF RESULTS

Table 4 provides the demographics of the population from which our sample was drawn. The table was provided by Twitter. 
Table 4: Demographics of population of TurkishWIN's Twitter handle
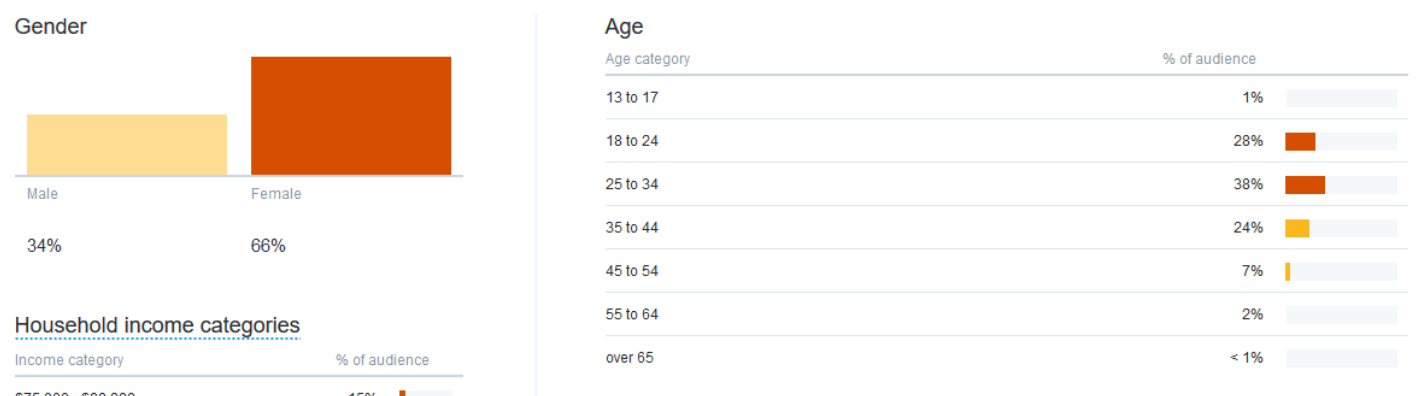

Household income categories

\begin{tabular}{|c|c|}
\hline Income category & $\%$ of audience \\
\hline$\$ 75,000-\$ 99,999$ & $15 \%$ | \\
\hline$\$ 175,000$ - $\$ 199,999$ & $12 \%$ I \\
\hline$\$ 125,000-\$ 149,999$ & $12 \%$ \\
\hline$\$ 150,000-\$ 174,999$ & $12 \%$ \\
\hline$\$ 250,000+$ & $12 \%$ \\
\hline
\end{tabular}
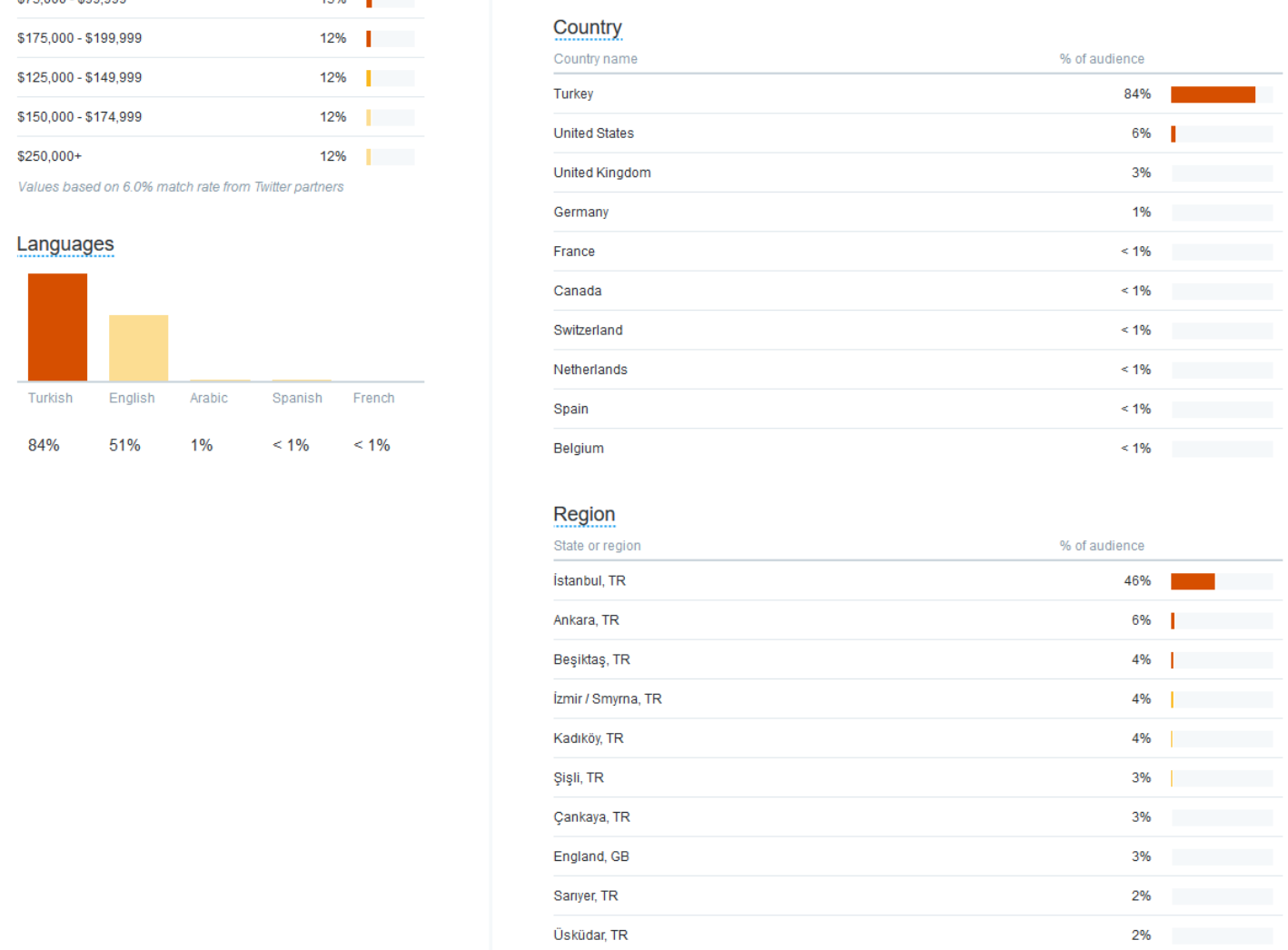

Table 5 shows the Model Summary of the 9 steps of the stepwise regression. At each step, a variable entered the model. There were no "deletion" steps. 
Table 5: Stepwise-regression model summary

Model Summary

\begin{tabular}{|c|c|c|c|c|}
\hline Model & $\mathrm{R}$ & R Square & Adjusted R Square & $\begin{array}{l}\text { Std. Error of the } \\
\text { Estimate }\end{array}$ \\
\hline 1 & $.376^{a}$ & .141 & .141 & .013868300900 \\
\hline 2 & $.502^{b}$ & .252 & .251 & .012946425600 \\
\hline 3 & $.533^{c}$ & .284 & .282 & .012674441700 \\
\hline 4 & $.545^{\mathrm{d}}$ & .297 & .296 & .012556100700 \\
\hline 5 & $.556^{\mathrm{e}}$ & .309 & .307 & .012456584600 \\
\hline 6 & $.560^{\mathrm{f}}$ & .314 & .311 & .012416980300 \\
\hline 7 & $.562^{\mathrm{g}}$ & .316 & .313 & .012399057100 \\
\hline 8 & $.565^{h}$ & .319 & .315 & .012376932300 \\
\hline 9 & $.568^{\mathrm{i}}$ & .323 & .319 & .012347580700 \\
\hline
\end{tabular}

a. Medium

b. Medium, Mentions

c. Medium, Mentions, Language

d. Medium, Mentions, Language, Evening

e. Medium, Mentions, Language, Evening, Saturday

f. Medium, Mentions, Language, Evening, Saturday, Sunday

g. Medium, Mentions, Language, Evening, Saturday, Sunday, Hashtags

h. Medium, Mentions, Language, Evening, Saturday, Sunday, Hashtags, Links

i. Medium, Mentions, Language, Evening, Saturday, Sunday, Hashtags, Links, Length

We can see that 9 variables entered the stepwise regression models, and, as noted earlier, none were deleted - all "survived." Table 6 provides the ANOVA table for the final (step 9) model.

Table 6: ANOVA Table results for final step of stepwise regression analysis

SSQ DF MSQ $\quad$ F $\quad$ SIG.

\begin{tabular}{|l|l|c|c|c|c|c|}
\hline & Regression & .107 & 9 & .01189 & 77.8 & .000 \\
\cline { 2 - 6 } Residual & .224 & 1469 & .00015 & & \\
\cline { 2 - 6 } Total & .331 & 1478 & & & \\
\hline
\end{tabular}

Our final piece of output is in Table 7, and displays the "coefficients table" for the last (9th) step of the stepwise-regression analysis (Engagement Rate as the dependent variable.) 
Table 7: Coefficients table for last step of the stepwise-regression analysis Unstandardized coeff. Standardized

\begin{tabular}{|l|l|c|c|c|c|c|}
\hline \multicolumn{2}{c}{} & \multicolumn{2}{c}{ B } & \multicolumn{2}{c}{ Std. Error } & \multicolumn{2}{c}{ Beta } & \multicolumn{2}{c}{ Sig. } \\
\hline & (Constant) & .021 & .002 & & 10.497 & .000 \\
\cline { 2 - 7 } & Medium & .013 & .001 & .373 & 16.914 & .000 \\
\cline { 2 - 7 } & Mentions & .003 & .000 & .272 & 11.212 & .000 \\
\cline { 2 - 7 } & Language & -.005 & .001 & -.167 & -7.542 & .000 \\
\cline { 2 - 7 } & Evening & .006 & .001 & .107 & 4.836 & .000 \\
\cline { 2 - 7 } & Saturday & .006 & .001 & .110 & 4.964 & .000 \\
\cline { 2 - 7 } & Sunday & .006 & .002 & .068 & 3.148 & .002 \\
\cline { 2 - 7 } & Hashtags & -.001 & .000 & -.074 & -3.098 & .002 \\
\cline { 2 - 7 } & Link & -.002 & .001 & -.075 & -3.134 & .002 \\
\cline { 2 - 7 } & Length & .000 & -.066 & -2.828 & .005 \\
\hline
\end{tabular}

As can be seen from Table 5, the R-squared for the final model is 0.323 ; in other words, (we estimate that) 32.3 percent of the variability in engagement rate can be explained by its linear relationship with the 9 variables that survived the stepwise-regression process. As noted earlier, the engagement rate measures the quality of the tweet, which, likely, is mostly driven by the emotional factors that we can't analyze with the available data. These emotional factors include: point of the view of the users reading the content, relationship with the tweet owner, the mood of the users at the moment they see the tweet, etc. Therefore, one can make an argument, that to explain over $30 \%$ of the variability in engagement rate, without any data on these emotional factors, is a considerable achievement.

We now discuss the 9 variables in our final stepwise-regression model: whether including a medium, the number of mentions, the language used in the tweet, whether the tweet was sent in the evening, whether the tweet was sent on a Saturday, whether the tweet was sent on a Sunday, the number of hashtags, whether the tweet included a link to another site, and the length of the tweet text without URLs. When we discuss coefficients, we always are referring to the change in engagement rate, holding the other 8 variables in the model constant; in selected explanations, we explicitly remind the reader of this appropriate interpretation.

One inference is that the variable, medium, which entered first, is the variable, which by itself, is most predictive of engagement rate. It has a positive coefficient, implying, of course, that including a medium in the tweet increases the engagement rate (by an estimated .013 when holding the other variables in the final model constant; it happens to have the same coefficient of .013 [rounded to 3 digits] in a simple regression also.) This makes sense, because people may be more likely to click a tweet to see the image - therefore, to engage.

A second inference is that variables such as the time of the day and the day of the week have a bearing on engagement rate. The engagement rate has a positive relationship with each of the variables: Evening, Saturday, and Sunday. It is a coincidence that each of the three respective coefficients is .006 (again, to 3 digits.) So, the engagement rate is predicted to be .006 higher if the tweet is sent in the evening, compared to being sent at one of the other times; also, the engagement rate is predicted to be .006 higher on a Saturday and a Sunday, as compared to the average of other 5 days of the week. The common point of all three of these variables may be 
that these are times when people often rest, so they may pay more attention to a tweet during these particular periods of times.

The language of the tweet is another factor that shows up as affecting the engagement rate. In our research, we defined a dummy variable coding English as 1, and Turkish is 0. The coefficient of this variable is negative. This is likely related to the demographics of the followers. In this case, as seen in Table 4, 84 percent of the followers of the Twitter handle we analyzed are Turkish speakers, while only the 51 percent of the followers are English speakers. Hence, it makes sense that the followers are more likely to engage with the tweets written in Turkish than in English. There is likely nothing "special" about Turkish, as opposed to the more general language point that tweets should be easy to read and understand if they are going to get people's attention and generate engagements. This happens in the most natural way if the tweet speaks "the same language" as the followers.

The number of mentions also has a positive relationship (i.e., positive coefficient) with the engagement rate. This may be a spontaneous instinct, since, when Twitter handles are mentioned, it is natural that the reader will click, like, or re-tweet the original tweet in which they have been mentioned - thus, engaging with the tweet.

When it comes to the number of hashtags, the situation is somewhat different. The hashtag is the most characteristic feature of Twitter; it connects tweets by making words clickable by simply putting the hashtag sign (\#) at the beginning of the word. Twitter is known by its hashtags. Most of the blog posts suggest to use the appropriate hashtags [5]. However, in our case, the number of hashtags has a negative relationship with the engagement rate, which means that when we increase the number of hashtags (holding all other 8 variables in the model constant), we see a lower engagement rate.

The length of the tweet is another factor that has a negative relationship with the engagement rate (again, holding the other 8 variables in the model constant.) Although there is a 140character limit, the longer the tweet, the lower the engagement rate is. This would seem to make intuitive sense.

Finally, including a link (URL) of another website also has a negative relationship to the engagement rate. This would seem to indicate that when they are checking their Twitter feed, people are not especially interested in clicking a link to go another website.

\section{Limitations and Directions for Future Research}

From January 1, 2016 to November 30, 2016, Twitter had 2 official announcements. The first announcement was about a change in its algorithm [6]. We note this, even though the change is unlikely have had any impact on this research.

In addition to that change, Twitter also has announced that they changed the 140-character limitation by not counting some of the attachments such as the rich media URLs and the Twitter handles replied [7]. As a result of this change, we may, on average, have slightly longer tweet texts in our data set.

Also, tweet followers' behavior may change from handle to handle and/or region to region. Hence, one may wish to view the results of our study as specific to the handle that we've analyzed. However, we believe that, by and large, our results will generalize to different handles and regions. We encourage, as a direction for future research, that our study be duplicated for other handles in other regions. 


\section{References}

Twitter. (n.d.). Number of monthly active Twitter users worldwide from 1st quarter 2010 to 3rd quarter 2016 (in millions). In Statista - The Statistics Portal. Retrieved December 7, 2016, from https://www.statista.com/statistics/282087/number-of-monthly-active-twitter-users/ http://twitter.com/turkishwin https://support.twitter.com/articles/166337\# https://support.twitter.com/articles/20171990\#

Price, Shalyla (2016, January 11). 6 Ways to Increase Twitter Engagement. Web log article. Retrieved December 7 , 2016 from https://www.socialmediaexaminer.com/6-ways-to-increase-twitter-engagement/

Jahr, Michael (2016, February 10). Never miss important Tweets from people you follow. Web log article. Retrieved December 7, 2016 from https://blog.twitter.com/2016/never-miss-important-tweets-from-peopleyou-follow

Sherman, Todd (2016, May 24). Coming soon: express even more in 140 characters. Web log article. Retrieved December 7, 2016 from https://blog.twitter.com/express-even-more-in-140-characters 